\title{
EVALUATION OF MAXILLARY SINUS VOLUME IN DENTATE, EDENTULOUS AND FREE END CASES AMONG A SAMPLE OF MALAY POPULATION USING ORTHOPANTOMOGRAM Belqees A. Allawa ${ }^{1}$, Nazih S. Mustafa ${ }^{1}$, Muhannad A. Kashmoola ${ }^{1}$ and Imad M. Al-Ani ${ }^{2}$
}

${ }^{1}$ Kulliyyah of Dentistry, International Islamic University Malaysia, Kuantan, Pahang, Malaysia. ${ }^{2}$ Department of Basic Medical Science, Kulliyyah of Medicine, International Islamic University Malaysia, Kuantan, Pahang, Malaysia.

Introduction: Maxillary sinus volume is important for dental surgery that involves the sinus or areas close to it due to the risk of oral antral communication.

Objective: The purpose of this study is to evaluate the maxillary sinus volumes in dentate, edentulous and free end cases among a sample of Malay population using orthopantomogram.

Methodology: Digital panoramic images were analysed using the software computer programs to measure the size of maxillary sinus in both sides by calculating the distance of the border of the sinus horizontally (width), vertically (length) and regional (height) then multiplying them to get the volume size in cm3. Images were divided into three cases (dentate cases, edentulous cases, free end cases).

Results: Of the 364 cases, 118 were dentate, 192 edentulous, and 54 free ends. There were differences in the maxillary sinus volumes between left and right sides in dentate cases, but not in edentulous and free end cases. There were no difference by gender while age was only significant among edentulous cases.

Conclusion: There is no relationship between the gender and volume in all cases. The volume increases with age in edentulous cases. The volume on the both left and right sides is similar in dentate but different in edentulous and free end cases.

\section{1-CHROMIUMETHYLENEDIAMINETETRAACETICACID ( ${ }^{51} \mathrm{Cr}$-EDTA) GLOMERULAR FILTRATION RATE (GFR) ANALYSIS: COMPARING TWO BLOOD SAMPLING WITH MULTIPLE BLOOD SAMPLING.}

Shahrir A. $\mathbf{R}^{1}$, Ibrahim Lutfi bin Shuaib ${ }^{2}$ and Sazilah binti Ahmad Sarji ${ }^{3}$

${ }^{1}$ Department of Radiology, Kulliyyah of Medicine, International Islamic University Malaysia. 25200 Kuantan, Pahang. ${ }^{2}$ Institut Perubatan dan Pergigian Termaju (Advanced Medical \& Dental Institute), Universiti Sains Malaysia Bertam, 13200 Kepala Batas, Pulau Pinang Malaysia. ${ }^{3}$ Department of Biomedical Imaging, Faculty of Medicine, University of Malaya, 50603 Kuala Lumpur, Malaysia

Introduction: The glomerular filtration rate (GFR) is the commonest method used to quantify renal function. Chromium-51-Ethylenediaminetetraaceticacid $\left({ }^{51} \mathrm{Cr}\right.$-EDTA) plasma clearance has been accepted as a gold standard in measuring GFR. Several methods have been proposed in which the glomerular filtration rate is estimated from using only one or two blood samples to using multiple blood samples.

Objective: The purpose of this study is to determine the accuracy of using two blood samples compared to multiple blood samples, for calculation of GFR by the ${ }^{51}$ Cr-EDTA method. In addition, the study aimed to determine the best combination time of two blood sampling and correlation with gender and ethnicity of the patients.

Methodology: ${ }^{51} \mathrm{Cr}$-EDTA tracer solution is injected into a vein in the upper arm. Six mL of whole blood is then drawn from the opposite arm at 120, 150, 180 and 240 minutes, centrifuged and $2 \mathrm{ml}$ aliquots of plasma are pipetted and counted for a period of 5 minute each time, in an automatic gamma well counter. GFR is calculated by measuring ${ }^{51} \mathrm{Cr}-$

7|Medical Research Symposium 2015 
EDTA plasma clearance using slope intercept method. The patients' height and weight is used to correct the GFR to body surface area. Correlations of the values are tested by Pearson and intra-class correlation, and mean and standard deviation derived from paired t-test.

Results: All time point combinations using two blood sampling shows a high correlation with multiple blood sampling method ( $\mathrm{p}$ value $<0.001, \mathrm{r}=0.909$ to 0.989 ). The best combination using two blood sampling is at 2 and 4 hour time point ( $r=0.989$ with 99\% consistency). There is no significant difference in the GFR obtained by two blood sampling and multiple blood sampling between gender and ethnicity.

Conclusion: ${ }^{51}$ Cr-EDTA GFR estimation using two blood sampling method is accurate and reliable. The two and four hour time point is found to be the best. Gender and ethnicity does not influence GFR estimation using two blood sampling method.

\section{A STUDY OF THE ARTERIAL VARIATIONS IN THE POPLITEAL REGIONS, ARE THEY SYMMETRICAL OR NOT? \\ Ahmad Ruzain Bin Salehuddin', Zunariah Binti Buyong ${ }^{2}$, Siti Kamariah Binti Che Mohamed ${ }^{3}$ and Radhiana Binti Hassan ${ }^{3}$}

${ }^{1}$ Anatomy Unit, Basic Medical Sciences Cluster, Faculty Of Medicine, University Teknologi Mara (Uitm) Sungai Buloh Campus, Jalan Hospital, 47000 Sungai Buloh, Selangor, Malaysia. ${ }^{2}$ Department of Basic Medical Sciences, Kulliyyah (Faculty) of Medicine, International Islamic University Malaysia, 25200 Kuantan, Pahang, Malaysia. ${ }^{3}$ Department of Radiology, Kulliyyah (Faculty) of Medicine, International Islamic University Malaysia, 25200 Kuantan, Pahang, Malaysia

Objective: To assess the prevalence of the arterial variations in the popliteal regions and the symmetrical characteristic of the popliteal artery branching patterns in both knees of all subjects.

Methodology: 77 subjects (154 knees) were prospectively studied using colour Doppler ultrasonography in this descriptive and cross-sectional study. No subject had anomalies of the lower limbs or history of previous lower limb surgery. Subjects with cardiovascular disease were excluded from this study.

Results: Prevalence of the popliteal artery variations was $4.5 \%$. The two variants (type I-B and type II-A) were seen in both knees. There were 5 subjects who had at least one variant in either side of their knees. Two subjects had type II-A occurred bilaterally in both knees while 3 other subjects had unilateral pattern of type I-B in combination with type I-A in their knees.

Conclusion: Symmetrical and non-symmetrical pattern can be seen in the population depending on the popliteal artery branching pattern.

\section{Review}

\section{REVISITING ESSENTIAL HYPERTENSION, REGULATION OF BLOOD PRESSURE CONTROL AND THE ADRENALINE HYPOTHESIS}

Aszrin Abdullah ${ }^{1}$, Azarisman Shah Mohd Shah ${ }^{2}$, Noriah Mohd Nor ${ }^{3}$ and Tariq Abdul Razak ${ }^{3}$

${ }^{1}$ Department of Basic Medical Sciences, Kulliyyah of Medicine. ${ }^{2}$ Department of Internal Medicine, Kulliyyah of Medicine.

${ }^{3}$ Department of Basic Medical Sciences, Kulliyyah of Pharmacy

Hypertension or high blood pressure, is a condition that has a significant impact on the world's population morbidity and mortality. The World Health Report 2002, stated that high blood pressure caused 7.1 million deaths throughout the world, which was approximately $13 \%$ of total deaths worldwide. The body's arterial blood pressure control depends on short-term and long-term mechanisms. Short-term BP regulation is mediated by the autonomic nervous system (ANS) targeting the heart, vessels, and adrenal medulla. The sympathetic nervous systems (SNS) crucial involvement in stressful conditions and short term regulation of blood pressure is also widely accepted. There has also been increasing

8|Medical Research Symposium 2015 